\title{
Pelvic Lymph Node Metastases in Prostate Cancer: Preoperative Detection With Dynamic Contrast-Enhanced Magnetic Resonance Imaging Compared With Postoperative Pathologic Result of Pelvic Lymph Node Dissection
}

\author{
Hoyoung Ryu', Byeongdo Song ${ }^{2}$, Jinho Hwang ${ }^{2}$, Sung Kyu Hong ${ }^{2}$, \\ Seok-Soo Byun ${ }^{2}$, Sang Eun Lee ${ }^{2}$, Sangchul Lee \\ ${ }^{1}$ Department of Urology, Seoul National University Hospital, Seoul, Korea \\ ${ }^{2}$ Department of Urology, Seoul National University Bundang Hospital, Seongnam, Korea
}

\begin{abstract}
Purpose: The aim of this study is evaluating the accuracy of preoperative magnetic resonance imaging (MRI) in patients who underwent pelvic lymph node dissection (PLND).

Materials and Methods: The medical records of 1,528 patients who underwent radical prostatectomy and PLND from 2003 to 2017 in Seoul National University Bundang Hospital were retrospectively reviewed. We evaluated the various clinicopathologic variables including preoperative MRI findings and pathologic lymph node (LN) metastasis. The prediction model for pathologic $\mathrm{LN}$ metastasis was assessed using univariate and multivariable logistic regression analyses and areas under receiver operating characteristic (ROC) curves.

Results: The mean age of our cohort was $66.4 \pm 6.7$ years. Positive LN finding of preoperative MRI finding was observed in $9.4 \%$ (145 of 1,528) of patients. $5.3 \%$ (81 of 1,528) of patients had confirmed final pathologic LN metastases. Sensitivity and specificity of preoperative MRI were $30.8 \%$ and $91.7 \%$, respectively. Multivariable analysis showed that preoperative MRI findings, clinical stage and biopsy Gleason score were independent significant predictors for pathologic LN metastasis $(p<0.001, p=0.002$, and $p<0.001$, respectively). Prediction model using preoperative MRI findings and National Comprehensive Control Network risk stratification showed fair accuracy using ROC analysis.

Conclusions: Preoperative MRI findings for pathologic LN metastasis showed limited prediction value. A large-scale, multicenter, prospective study is needed to fully evaluate the clinical significance of preoperative MRI. (Korean J Urol Oncol 2017;15:158-164)
\end{abstract}

Key Words: Prostate $\cdot$ Radical prostatectomy $\cdot$ Magnetic resonance imaging $\cdot$ Lymph nodes

Received October 20, 2017, Revised October 23, 2017,

Accepted October 23, 2017

Corresponding Author: Byeongdo Song

Department of Urology, Seoul National University Bundang Hospital, 82 Gumi-ro 173beon-gil, Bundang-gu, Seongnam 13620, Korea

E-mail: uromedi @naver.com

Tel: +82-31-787-7345, Fax: +82-31-787-4057

ORCID code: https://orcid.org/0000-0003-3961-1258

- This research was supported by a grant of the Korean Health Technology R\&D Project through the Korea Health Industry Development Institute (KHIDI), funded by the Ministry of Health \& Welfare, Republic of Korea (grant number: HI17C1264).

\section{INTRODUCTION}

Prostate cancer is the most common cancer in men and the third most common cause of death among malignancies in male population. ${ }^{1}$ For localized prostate cancer, definite treatments such as radical prostatectomy (RP) or radiation therapy could be primary therapeutic options. However, once prostate cancer invades lymph node or other organs, therapeutic effects of such

(c) (F) This is an Open Access article distributed under the terms of the Creative Commons Attribution Non-Commercial License (http://creativecommons.org/licenses/by-nc/4.0/) which permits unrestricted non-commercial use, distribution, and reproduction in any medium, provided the original work is properly cited.

2017 (C) Copyright The Korean Urological Oncology Society and The Korean Prostate Society. All Rights Reserved. 
treatment modalities become limited. Even if the therapeutic role of pelvic lymph node dissection (PLND) in patients with low-risk prostate cancer is still an open question, patients with intermediate- to high-risk disease are likely to benefit from PLND. ${ }^{2}$ For this reason, early detection of lymph node invasion (LNI) is important for treatment of prostate cancer.

The Partin tables, ${ }^{3}$ the National Comprehensive Control Network (NCCN) practice guidelines nomogram, ${ }^{4,5}$ the D'Amico risk-classification ${ }^{6,7}$ are commonly used to assess the risk of prostate cancer. Such risk assessment tools use preoperative variables including prostate-specific antigen (PSA), biopsy Gleason score, and clinical stage to predict prognosis of LNI and prostate cancer after treatments.

However, such preoperative assessing tools do not include preoperative image studies including magnetic resonance imaging (MRI). Recently, the multiparacentric MRI (mp-MRI), which is the combination of conventional T1-weighted MRI (T1WI) and T2-weighted MRI (T2WI) with various functional imaging modalities including diffusion-weighted MRI (DWI), dynamic contrast enhanced MRI (DCEI), and magnetic resonance spectroscopy, has been applied in clinical filed. ${ }^{8}$ The role of mp-MRI has been expanded broadly to prostate biopsy, active surveillance, advanced disease detection, and local recurrence detection after RP. ${ }^{9}$ However, the diagnostic value of imaging studies including MRI are still debatable. Wolf et al. ${ }^{10}$ reported that CT and MRI showed high specificity of $97 \%$, but relatively low sensitivity of $36 \%$. They concluded that nodal imaging studies should only be recommended for patients having a probability of $45 \%$ or higher for LNI. Min et al. ${ }^{11}$ reported that a combined approach of T1W, T2W, and DCEI with DWI demonstrated an accurate detection rate of prostate cancer, showing high specificity but relatively lower sensitivity. Hövels et al. ${ }^{12}$ reported MRI demonstrated an equally poor performance in the detection of LNIs from prostate cancer through meta-analysis.

The aim of this study is demonstrating the predictive power of preoperative MRI for LNI in prostate cancer patients who underwent PLND.

\section{MATERIALS AND METHODS}

With an approval from Institutional Review Board (B-1710-427-110), the medical records of 3,379 patients who underwent RP for prostate cancer at Seoul National University
Bundang Hospital from November 2003 to May 2017 were analyzed retrospectively. After excluding patients who did not undergo PLND and those with missing data about lymph node state, 1,528 patients were included in our analyses. We evaluated the various clinicopathologic variables including preoperative MRI and pathologic LNI.

In our institution, every patient diagnosed with prostate cancer underwent preoperative prostate MRI, including T1WI, T2WI, DWI, and DCEI. Positive lymph node enlargement (LNE) on MRI was defined when increased over $10 \mathrm{~mm}$.

We stratified the cohorts into 3 groups according to the number of LNE: no significant LNE group, one LNE group, and more than 2 LNE group. Each group was graded as 0, 1, and 2, respectively.

Along with preoperative MRI, we used the NCCN risk stratification to predict diagnostic values for metastasis. In NCCN risk stratification system, there are 5 risk categories: very low (T1c and Gleason score $\leq 6$ and PSA $<10 \mathrm{ng} / \mathrm{mL}$ and $<3$ positive core biopsies); low (T1-T2a and Gleason score $\leq 6$ and PSA $<10 \mathrm{ng} / \mathrm{mL})$; intermediate $(\mathrm{T} 2 \mathrm{~b}-\mathrm{T} 2 \mathrm{c}$ or Gleason score 7 or $\mathrm{PSA}=10^{-20} \mathrm{ng} / \mathrm{mL}$ ); high (T3a or Gleason score $8-10$ or PSA $>20 \mathrm{ng} / \mathrm{mL}$ ); and very high (T3b-T4 or Gleason primary pattern 5). ${ }^{13}$ The entire cohorts were classified into 3 categories as followings: low-risk group (very low-risk and low-risk group), intermediate-risk group, and high-risk group (high-risk and very high-risk group) according to NCCN risk stratification, with each group graded as $0,1,2$, respectively. To assess predictive power of combination of preoperative MRI and NCCN risk stratification, we added the numerical grades of MRI group $(0,1$, and 2$)$ and NCCN risk group (0, 1, and 2).

The prediction model for pathologic lymph node metastasis was evaluated using univariate and multivariable logistic regression analyses. Areas under receiver operating characteristic (ROC) curves (AUC) was used as an index of diagnostic accuracy.

IBM SPSS Statistics ver. 19.0 (IBM Co., Armonk, NY, USA) was used for statistical analysis, and ROC curve and the prediction factors were estimated using MEDCALC ver. 12 (MedCalc, Ostend, Belgium). All p-values were 2-sided, and $\mathrm{p}$ $<0.05$ was considered as a significant result.

\section{RESULTS}

Demographic characteristics of subjects were as shown in 
Table 1. The mean age of the cohort was $66.4 \pm 6.7$ years. Median concentration of serum PSA was $11.34 \mathrm{ng} / \mathrm{dL}$ (interquartile range, 6.91-20.11 ng/dL). Positive preoperative MRI finding was observed in $9.4 \%$ (145 of 1,528) of patients. $5.3 \%$ (81 of 1,528 ) of patients had confirmed final pathologic lymph node metastases.

Multivariable analysis showed that preoperative MRI findings $(\mathrm{p}<0.001)$, clinical stage $(\mathrm{p}=0.001)$ and biopsy Gleason score $\geq 8(p<0.001)$ were significant independent predictors for

Table 1. Baseline characteristics of patients $(n=1,528)$

\begin{tabular}{|c|c|}
\hline Characteristic & Value \\
\hline Age $(\mathrm{yr})$ & $66.4 \pm 6.7(41-82)$ \\
\hline BMI $\left(\mathrm{kg} / \mathrm{m}^{2}\right)$ & $24.5 \pm 2.8(14.2-54.9)$ \\
\hline PSA (ng/dL) & $11.3(6.9-20.1)$ \\
\hline Volume of prostate $(\mathrm{g})$ & $35.8 \pm 14.2(9.3-116.0)$ \\
\hline \multicolumn{2}{|l|}{ Gleason biopsy grade } \\
\hline 6 & $299(19.6)$ \\
\hline 7 & $752(49.2)$ \\
\hline $8 \geq$ & $477(31.2)$ \\
\hline \multicolumn{2}{|l|}{ Clinical stage } \\
\hline T1c & $773(50.6)$ \\
\hline $\mathrm{T} 2 \mathrm{a}$ & $415(27.2)$ \\
\hline $\mathrm{T} 2 \mathrm{~b}+\mathrm{T} 2 \mathrm{c}$ & $54(3.5)$ \\
\hline$>\mathrm{T} 3$ & $286(18.7)$ \\
\hline \multicolumn{2}{|l|}{ Risk group } \\
\hline Low & $156(10.2)$ \\
\hline Intermediate & $617(40.4)$ \\
\hline High & $755(49.4)$ \\
\hline \multicolumn{2}{|l|}{ Operation method } \\
\hline RALP & $1,060(69.4)$ \\
\hline $\mathrm{RRP}$ & $458(30.0)$ \\
\hline LRP & $10(0.6)$ \\
\hline MRI LN positivity & $145 / 1,528(9.4)$ \\
\hline 1 & 126 \\
\hline 2 & 19 \\
\hline Mean PLND LN yield & $7.7 \pm 5.5(1-34)$ \\
\hline PLND LN positivity & $81 / 1,528(5.3)$ \\
\hline 1 & 43 \\
\hline 2 & 22 \\
\hline 3 & 11 \\
\hline 4 & 3 \\
\hline 5 & 1 \\
\hline 6 & 1 \\
\hline
\end{tabular}

Values are presented as mean \pm standard deviation (range), median (interquartile range), number (\%), or number.

BMI: body mass index, PSA: prostate specific antigen, RALP: robot-assisted laparoscopic radical prostatectomy, RRP: radical retropubic prostatectomy, LRP: laparoscopic radical prostatectomy, MRI: magnetic resonance imaging, LN: lymph node, PLND: pelvic lymph node dissection. pathologic LN metastasis (Table 2).

Preoperative mp-MRI (T1W, T2W, DWI, and DCEI) of prostate alone achieved a sensitivity of $30.86 \%$ and a specificity of $91.71 \%$, respectively. NCCN risk alone and the combination of preoperative mp-MRI and NCCN risk stratification achieved sensitivities of $90.12 \%$ and $99.45 \%$ respectively, and specificities of $52.87 \%$ and $49.38 \%$ respectively (Table 3 ).

Among 145 patients with positive LN on preoperative MRI, only 25 patients had confirmed final pathologic lymph node metastases. Especially, Among 126 patients with one positive LN on preoperative MRI, only 21 patients $(16.7 \%)$ had confirmed final pathologic lymph node metastasis (Table 4).

Through ROC analysis, prediction model using preoperative MRI findings alone showed AUC value of 0.613 (0.588-0.638). The addition of preoperative MRI findings to NCCN risk stratification showed fair accuracy, improving the AUC to 0.758 $(0.735-0.779)$ from 0.613 with NCCN risk stratification alone (Fig. 1).

\section{DISCUSSION}

There are several pretreatment prostate cancer risk stratification systems or nomograms based on prognostic power of initial PSA, biopsy Gleason score and clinical $\mathrm{T}$ stage including D'Amico risk stratification, NCCN practice guideline risk-stratification, and the D'Amico risk-classification. ${ }^{14}$

The Partin tables are the first nomogram to predict rates of organ-confined disease, positive margins, the risk of seminal vesicle and lymph node positivity after RP, which uses commonly available preoperative factors including serum PSA level, clinical stage and biopsy Gleason score. ${ }^{15}$ The Partin tables were initially established based on the information of the patients who underwent RP between 1982 and 1991, and it was upgraded with newer data acquired between 2006 to 2011 . $^{16}$ The predictive accuracy of the Partin tables for LNI was from $76 \%$ to $84 \% .{ }^{17-20}$ Recently, the Partin tables are commonly used for making therapeutic decision to perform a lymphadenectomy at the time of RP. The NCCN guideline risk stratification contains the minimum of clinical stage, grade and PSA it is applied for the selection of optimal therapeutic options as well as for the prediction of biochemical failure rate after definitive local therapy. ${ }^{4,21}$ NCCN practice guideline LNI nomogram is also used for predicting the prognosis of $\mathrm{LN}$ positive disease after lymphadenectomy. Moreover, the D'Amico risk-classification 
Table 2. Univariate and multivariable competing risks analysis evaluating of lymph node invasion and risk factors

\begin{tabular}{|c|c|c|c|c|c|c|}
\hline \multirow{2}{*}{ Variable } & \multicolumn{3}{|c|}{ Univariate analysis } & \multicolumn{3}{|c|}{ Multivariate analysis } \\
\hline & HR & $95 \% \mathrm{CI}$ & p-value & HR & $95 \% \mathrm{CI}$ & $\mathrm{p}^{\text {-value }}$ \\
\hline Age & 1.003 & $0.970-1.037$ & 0.882 & - & - & - \\
\hline Body mass index & 0.962 & $0.886-1.043$ & 0.347 & - & - & - \\
\hline Smoking amount (pack $\times$ year) & 0.997 & $0.984-1.010$ & 0.625 & - & - & - \\
\hline \multicolumn{7}{|l|}{ Operation methods } \\
\hline Open vs. robotic & 0.697 & $0.412-1.179$ & 0.179 & - & - & - \\
\hline Prostate volume on TRUS & 1.010 & $0.996-1.024$ & 0.179 & - & - & - \\
\hline $\mathrm{NCCN}$ risk group & 7.639 & $3.881-15.033$ & $<0.001$ & & & \\
\hline Low vs. intermediate & 1.779 & $0.217-14.564$ & 0.591 & - & - & - \\
\hline Low vs. high & 16.591 & $2.288-120.288$ & 0.005 & - & - & - \\
\hline PSA & 1.014 & $1.008-1.020$ & $<0.001$ & - & - & - \\
\hline \multicolumn{7}{|l|}{ Clinical stage } \\
\hline Stage T1c & - & - & - & - & - & - \\
\hline Stage T2 & 2.737 & $1.599-4.687$ & $<0.001$ & 2.516 & $1.448-4.370$ & 0.001 \\
\hline Stage $\geq \mathrm{T} 3$ & 7.661 & $4.160-14.107$ & $<0.001$ & 2.853 & $2.853-10.308$ & $<0.001$ \\
\hline \multicolumn{7}{|l|}{ Gleason score } \\
\hline 6 & - & - & - & - & - & - \\
\hline 7 & 3.596 & $0.782-16.531$ & 0.101 & 3.843 & $0.828-17.832$ & 0.085 \\
\hline$\geq 8$ & 13.998 & $3.410-57.467$ & $<0.001$ & 11.683 & $2.812^{-48.541}$ & 0.001 \\
\hline DRE & 2.798 & $1.782-4.391$ & $<0.001$ & & & \\
\hline MRI LNI positive & 3.375 & $2.266-5.027$ & $<0.001$ & 2.694 & $1.707-4.251$ & $<0.001$ \\
\hline
\end{tabular}

HR: hazard ratio, CI: confidence interval, TRUS: transrectal ultrasound, NCCN: National Comprehensive Control Network, PSA: prostatespecific antigen, DRE: digital rectal examination, MRI: magnetic resonance imaging, LNI: lymph node invasion.

Table 3. Sensitivity, specificity, and predictive values of each risk stratifications

\begin{tabular}{lccccc}
\hline \multicolumn{1}{c}{ Variable } & Sensitivity & Specificity & PPV & NPV & AUC (95\% CI) \\
\hline Preoperative MRI & 30.86 & 91.71 & 17.24 & 95.95 & $0.613(0.543-0.684)$ \\
NCCN & 90.12 & 52.87 & 9.67 & 98.97 & $0.717(0.671-0.763)$ \\
Preoperative MRI + NCCN & 99.45 & 49.38 & 33.33 & 94.92 & $0.758(0.707-0.809)$ \\
\hline
\end{tabular}

PPV: positive predictive value, NPV: negative predictive value, AUC: areas under receiver operating characteristic curves, CI: confidence interval, MRI: magnetic resonance imaging, NCCN: National Comprehensive Control Network.

was originally developed to predict biochemical recurrence in prostate cancer patients after treatment. ${ }^{6}$

These 3 tools have been applied frequently in routine clinical practice. When comparing these 3 tools for prediction of LNI, Abdollah et al. ${ }^{20}$ showed that NCCN guideline LNI nomogram (AUC 82\%) outperformed the Partin tables (73\%) and the D'Amico risk-classification (75\%).

However, among these 3 nomograms, imaging studies including preoperative MRI has not been accepted as essential parameter to predict LNI. Therefore, we investigated whether preoperative MRI could improve predictive power of conventional nomograms. This study aimed to evaluate the accuracy of preoperative MRI in patients who underwent RP with PLND and determinate whether preoperative MRI could improve the diagnostic value of conventional NCCN.

The advent of mp-MRI is known to provide more accurate mean of diagnosing prostate cancer in men with elevated PSA level, achieving a specificity of $90 \%$, a negative predictive valve of $85 \% .^{22,23}$ To achieve the highest sensitivity and specificity in detecting and assessing suspicious lesion within prostate, mp-MRI combines conventional T1WI and T2WI with various functional imaging modalities, with a minimum of 2 modalities. DWI and magnetic resonance spectroscopic imaging add specificity for lesion characterization, and DCE adds sensitivity for cancer detection. ${ }^{8}$

There are several guidelines from the European Association 
Table 4. Final lymph node pathology of patients who have positive lymph node findings on preoperative MRI

\begin{tabular}{crc}
\hline Positive LN on preoperative MRI & MRI LN=1 & MRI LN $\geq 2$ \\
\hline Negative pathologic LNI & $105(83.3)$ & $15(78.9)$ \\
Positive pathologic LNI & $21(16.7)$ & $4(21.1)$ \\
Pathologic LNI & & \\
0 & $105(83.3)$ & $15(78.9)$ \\
1 & $8(6.3)$ & $0(0)$ \\
2 & $7(5.6)$ & $2(10.5)$ \\
3 & $3(2.4)$ & $2(10.5)$ \\
4 & $2(1.6)$ & $0(0)$ \\
5 & $0(0)$ & $0(0)$ \\
6 & $1(0.8)$ & $0(0)$
\end{tabular}

Values are presented as number (\%).

MRI: magnetic resonance imaging, LN: lymph node, LNI: lymph node invasion.

of Urology (EAU), the American Urological Association (AUA), and the NCCN, mentioning the potential role of mp-MRI in prostate biopsy, active surveillance, and recurrent prostate cancer. ${ }^{9}$

The EAU guideline suggests that mp-MRI followed by transrectal ultrasonography (TRUS)-guided or MRI-guided biopsies might be useful to detect prostate cancer especially in anterior region, whereas conventional TRUS-guided biopsy is still performed more frequently instead of the corresponding newer modalities. However, mp-MRI is not recommended for the purpose of staging in case of low-risk prostate cancer, except when the results of MRI could influence management. ${ }^{24}$ The AUA guideline suggests that mp-MRI could identify local recurrence lesion and improve salvage radiation targeting, ${ }^{25}$ and the $\mathrm{NCCN}$ guideline suggests that mp-MRI could aid in deciding active surveillance, yet concluded mp-MRI is not recommended for routine use. ${ }^{26}$

In detecting lymph node metastasis, mp-MRI showed a high specificity of $91 \%-97 \%$ but a low sensitivity of $14 \%-$ $36 \%{ }^{10,11,27}$ Hövels et al. ${ }^{12}$ mentioned poor performance of MRI in detection of lymph node metastasis through meta-analysis study. Because of such low sensitivity, mp-MRI alone was not recommended for lymph node workup in patients without suspicious lymph node lesions on $\mathrm{CT}^{28}$ To evaluate predictive power of preoperative MRI for LNI, we analyzed the medical records of 1,528 patients who underwent RP with PLND, which is the largest cohort as far as we know.

In this study, the mp-MRI showed a high specificity of $91.7 \%$ and relatively a low sensitivity of $30.8 \%$, which are sim-

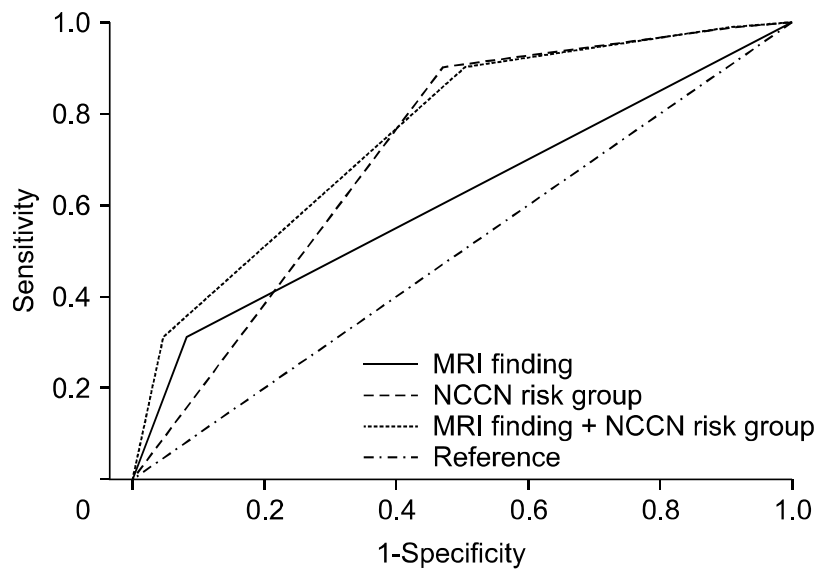

Fig. 1. Receiver operating characteristic curves: prediction model using preoperative MRI findings and NCCN risk stratification. MRI: magnetic resonance imaging, NCCN: National Comprehensive Control Network.

ilar to the previous study results. The ROC curve of preoperative MRI alone showed AUCs of 0.613 for lymph node metastasis. ROC curve of NCCN nomogram showed AUCs of 0.713 that was similar to the results of Abdollah et al. ${ }^{20}$.

When combining risk grades of NCCN nomogram with the outcomes of preoperative MRI, the specificity and the sensitivity were $99.5 \%$ and $49.4 \%$, respectively. The ROC curve showed AUCs of 0.782 . When the corresponding 2 parameters were combined, the AUCs became smaller than 0.9, but the value was greater compared to 0.613 and 0.713 of single parameters.

There are several limitations in this study. At first, while the large number of cohort enabled us to detect significant differences, our database is derived from the retrospective records of patients who were treated at single institution. Therefore, multi-institutional studies might be needed to confirm our findings. Furthermore, we were unable to match and compare the locations of lymph node lesions shown in preoperative MRI with the actual pathologically confirmed LNI sites. Moreover, the lack of information on long-term survival including cancer-specific survival or overall survival could be another limitation to our study.

\section{CONCLUSIONS}

Our results show that preoperative MRI findings for pathologic LN metastasis alone showed limited prediction value. However, when combining with conventional NCCN risk 
nomogram, preoperative MRI could improve predictive power. A large-scale, multicenter, and prospective study is needed to fully evaluate the clinical significance of preoperative MRI.

\section{CONFLICT OF INTEREST}

The authors claim no conflicts of interest.

\section{REFERENCES}

1. Siegel RL, Miller KD, Jemal A. Cancer statistics, 2017. CA Cancer J Clin 2017;67:7-30.

2. Joung JY, Cho IC, Lee KH. Role of pelvic lymph node dissection in prostate cancer treatment. Korean J Urol 2011;52: 437-45.

3. Makarov DV, Trock BJ, Humphreys EB, Mangold LA, Walsh PC, Epstein JI, et al. Updated nomogram to predict pathologic stage of prostate cancer given prostate-specific antigen level, clinical stage, and biopsy Gleason score (Partin tables) based on cases from 2000 to 2005. Urology 2007;69:1095-101.

4. NCCN Clinical Practice Guidelines in Oncology (NCCN Guidelines ${ }^{\circledR}$ ). Prostate Cancer Version 3 [Internet]. Fort Wathington (PA): National Comprehensive Cancer Network; 2016 [cited on 2017 Jan 2]. Available from: https://www.tri-kobe.org/nccn/ guideline/urological/english/prostate.pdf.

5. Cagiannos I, Karakiewicz P, Eastham JA, Ohori M, Rabbani $\mathrm{F}$, Gerigk C, et al. A preoperative nomogram identifying decreased risk of positive pelvic lymph nodes in patients with prostate cancer. J Urol 2003;170:1798-803.

6. D'Amico AV, Whittington R, Malkowicz SB, Schultz D, Blank K, Broderick GA, et al. Biochemical outcome after radical prostatectomy, external beam radiation therapy, or interstitial radiation therapy for clinically localized prostate cancer. JAMA 1998;280:969-74.

7. Boorjian SA, Karnes RJ, Rangel LJ, Bergstralh EJ, Blute ML. Mayo Clinic validation of the D'amico risk group classification for predicting survival following radical prostatectomy. J Urol 2008;179:1354-60.

8. Barentsz JO, Richenberg J, Clements R, Choyke P, Verma S, Villeirs G, et al. ESUR prostate MR guidelines 2012. Eur Radiol 2012;22:746-57.

9. Yoo S, Kim JK, Jeong IG. Multiparametric magnetic resonance imaging for prostate cancer: a review and update for urologists. Korean J Urol 2015;56:487-97.

10. Wolf JS Jr, Cher M, Dall'era M, Presti JC Jr, Hricak H, Carroll PR. The use and accuracy of cross-sectional imaging and fine needle aspiration cytology for detection of pelvic lymph node metastases before radical prostatectomy. J Urol 1995;153(3 Pt 2):993-9.

11. Min BD, Kim WT, Cho BS, Kim YJ, Yun SJ, Lee SC, et al. Usefulness of a combined approach of T1-weighted, T2-weighted, dynamic contrast-enhanced, and diffusion-weighted imaging in prostate cancer. Korean J Urol 2012;53:830-5.

12. Hövels AM, Heesakkers RA, Adang EM, Jager GJ, Strum S, Hoogeveen YL, et al. The diagnostic accuracy of CT and MRI in the staging of pelvic lymph nodes in patients with prostate cancer: a meta-analysis. Clin Radiol 2008;63:387-95.

13. Mohler JL, Armstrong AJ, Bahnson RR, D'Amico AV, Davis BJ, Eastham JA, et al. Prostate cancer, version 1.2016. J Natl Compr Canc Netw 2016;14:19-30.

14. Rodrigues G, Warde P, Pickles T, Crook J, Brundage M, Souhami L, et al. Pre-treatment risk stratification of prostate cancer patients: a critical review. Can Urol Assoc J 2012;6: 121-7.

15. Partin AW, Kattan MW, Subong EN, Walsh PC, Wojno KJ, Oesterling JE, et al. Combination of prostate-specific antigen, clinical stage, and Gleason score to predict pathological stage of localized prostate cancer. A multi-institutional update. JAMA 1997;277:1445-51.

16. Eifler JB, Feng Z, Lin BM, Partin MT, Humphreys EB, Han $\mathrm{M}$, et al. An updated prostate cancer staging nomogram (Partin tables) based on cases from 2006 to 2011. BJU Int 2013;111:22-9.

17. Blute ML, Bergstralh EJ, Partin AW, Walsh PC, Kattan MW, Scardino PT, et al. Validation of Partin tables for predicting pathological stage of clinically localized prostate cancer. J Urol 2000;164:1591-5.

18. Yu JB, Makarov DV, Sharma R, Peschel RE, Partin AW, Gross CP. Validation of the partin nomogram for prostate cancer in a national sample. J Urol 2010;183:105-11.

19. Penson DF, Grossfeld GD, Li YP, Henning JM, Lubeck DP, Carroll PR. How well does the Partin nomogram predict pathological stage after radical prostatectomy in a community based population? Results of the cancer of the prostate strategic urological research endeavor. J Urol 2002;167:1653-7.

20. Abdollah F, Schmitges J, Sun M, Thuret R, Djahangirian O, Tian Z, et al. Head-to-head comparison of three commonly used preoperative tools for prediction of lymph node invasion at radical prostatectomy. Urology 2011;78:1363-7.

21. D'Amico AV, Whittington R, Malkowicz SB, Fondurulia J, Chen MH, Kaplan I, et al. Pretreatment nomogram for prostate-specific antigen recurrence after radical prostatectomy or external-beam radiation therapy for clinically localized prostate cancer. J Clin Oncol 1999;17:168-72.

22. Thompson J, Lawrentschuk N, Frydenberg M, Thompson L, Stricker P; USANZ. The role of magnetic resonance imaging in the diagnosis and management of prostate cancer. BJU Int 2013;112 Suppl 2:6-20.

23. Raz O, Haider M, Trachtenberg J, Leibovici D, Lawrentschuk N. MRI for men undergoing active surveillance or with rising PSA and negative biopsies. Nat Rev Urol 2010;7:543-51.

24. Heidenreich A, Bastian PJ, Bellmunt J, Bolla M, Joniau S, 
van der Kwast $\mathrm{T}$, et al. EAU guidelines on prostate cancer. Part II: Treatment of advanced, relapsing, and castration-resistant prostate cancer. Eur Urol 2014;65:467-79.

25. Thompson IM, Valicenti RK, Albertsen P, Davis BJ, Goldenberg SL, Hahn C, et al. Adjuvant and salvage radiotherapy after prostatectomy: AUA/ASTRO Guideline. J Urol 2013;190:441-9.

26. Mohler JL, Kantoff PW, Armstrong AJ, Bahnson RR, Cohen M, D'Amico AV, et al. Prostate cancer, version 2.2014. J Natl Compr Canc Netw 2014;12:686-718.
27. Jeong IG, Lim JH, You D, Kim MH, Choi HJ, Kim JK, et al. Incremental value of magnetic resonance imaging for clinically high risk prostate cancer in 922 radical prostatectomies. J Urol 2013;190:2054-60.

28. Van den Bergh L, Lerut E, Haustermans K, Deroose CM, Oyen R, Isebaert $S$, et al. Final analysis of a prospective trial on functional imaging for nodal staging in patients with prostate cancer at high risk for lymph node involvement. Urol Oncol 2015;33:109.e23-31. 